\title{
Jumping Risk Communities in the Energy Industry: An Empirical Analysis Based on Time-Varying Complex Networks
}

\author{
Hui Wang $\mathbb{D},{ }^{1}$ Lili Jiang $\mathbb{D},{ }^{2}$ Hongjun Duan $\mathbb{D},{ }^{3}$ Yifeng Wang $\mathbb{D},{ }^{2}$ Yichen Jiang $\mathbb{D}^{4}$, \\ and Xiaolei Zhang $\mathbb{D}^{1}$ \\ ${ }^{1}$ Pan-Asia Business School, Yunnan Normal University, Kunming, China \\ ${ }^{2}$ Business School, Suqian College, Suqian, China \\ ${ }^{3}$ Jiangsu Vocational and Technical College of Finance and Economics, Huaian, China \\ ${ }^{4}$ Zhejiang Science and Technology Information Research Institute, Hangzhou, China
}

Correspondence should be addressed to Lili Jiang; 719821098@qq.com, Yichen Jiang; beaconspy@qq.com, and Xiaolei Zhang; financialmath@163.com

Received 9 May 2021; Revised 7 December 2021; Accepted 16 December 2021; Published 11 January 2022

Academic Editor: M. M. El-Dessoky

Copyright @ 2022 Hui Wang et al. This is an open access article distributed under the Creative Commons Attribution License, which permits unrestricted use, distribution, and reproduction in any medium, provided the original work is properly cited.

\begin{abstract}
This paper uses the 5-five-minute high-frequency data of energy-listed companies in China's A-share market to extract the jump of energy stock prices and build a dynamic stock price jump complex network. Then, we analyze the clustering effect of the complex network. The research shows that the energy stock price jump is an important part of stock price volatility, and the complex network of energy stock jump risk has obvious time-varying characteristics. However, the infection problem of stock price jump risks needs specific analysis. China's coal industry has an important influence on the development of China's energy industry. According to the clustering analysis results of the network community, the clustering effect of the network community has time-varying characteristics. After October 2017, the clustering effect of the jumping risk of the coal industry and the new energy industry is obvious. The risk contagion within the new energy industry community is a key point for the development of the new energy industry.
\end{abstract}

\section{Introduction}

With the acceleration of global economic integration, the energy finance market developed based on the energy industry and relying on the financial market has become an important global financial trading platform. The effective combination of the energy and financial markets has become the key to whether the energy market can meet the growing energy demand in the human economy. At the same time, with the rapid development of energy finance, energy and financial risk management has become the key to the development of the international energy and international financial markets. The energy finance market efficiently allocates resources while spreading the risks of the energy finance market throughout the industry. This paper takes the energy stock price in China's stock market as the research object and uses the realized jump method to measure the jump risk in the energy financial market. To study the dynamic characteristics of a complex network of energy prices, we use Prim's algorithm to build a complex network of energy price jumping risks.

The main work of our paper is as follows. First, we use the five-minute high-frequency data of the energy stock market to obtain the realized volatility. Second, we use the realized jump method to obtain the jump risk of the energy stock. Third, we build complex networks for all stock price jumps to study the dynamic changes of complex networks. Fourth, the community method is used to study the dynamic changes in the energy stock jump communities. Through our research, the paper draws the following conclusions. (1) The energy stock price jump is an important part of energy stock price volatility. (2) The complex network of the energy company stock price jump has obvious time-varying characteristics. After October 
2017, the link between the stock price jump is closer, and the risk of a stock price jump is more likely to occur. (3) The energy stock complex network community has a strong time-varying feature. (4) China's coal industry is the most critical in the complex network of the entire energy industry, and the internal risk management of the coal industry is very important. (5) The risk contagion within the new energy industry community is vital for the development of the new energy industry.

The main contributions of this paper are as follows. (1) This paper takes the jump of the energy industry stock price as the object and studies the jumping risk problem of the energy industry by using the complex network method. (2) This paper analyzes the dynamic characteristics of the complex network of energy stock jump risk. (3) The coal industry occupies a central position in China's energy complex network, and the risk of jumping within the coal industry community and the new energy industry community is worthy of attention.

\section{Literature Review}

Energy finance market risk management has always been a hot issue for many scholars around the world. Many scholars have studied the impact of financial markets on energy and financial markets from the perspectives of the stock, gold, and foreign exchange markets. Kaneko and Lee [1] found a negligible interaction between oil prices and stock returns by studying the relationship between the oil market and the stock market. Basher and Sadorsky [2] used a multifactor risk analysis model to investigate the relationship between oil price risk and emerging stock market returns using conditional regression and unconditional regression. It found that oil price volatility significantly influences stock market returns in emerging markets. The results of Zhang et al. [3] show a significant spillover effect between oil prices and the US dollar exchange rate. Narayan [4] showed a longterm cointegration relationship among international gold prices, future oil prices, and spot prices. The change in oil price affects mainly the price of gold through inflation.

The above studies are based on the analysis of energy market prices, and volatility is an important indicator to measure risk. This paper will use realized volatility and the realized jump method to study the complex network of energy stock price jumps; therefore, it is necessary to review the related research.

With continuous research on volatility, the use of historical standard deviation to study volatility can no longer meet the needs of modern research. The method of extracting realized volatility from high-frequency data has attracted increasing attention from scholars, such as Andersen and Bollerslev [5] and Barndorff-Nielsen and Shephard [6]. It is precise because of the rapid development of realized volatility methods that scholars can use realized methods to separate realized jumps from realized volatility so that they can more accurately study the relevant properties of price jumps. For example, Wang et al. [7], Anderson et al. [8], Zeng and Zuo [9], Xu et al. [10], Wang [7], and $\mathrm{Hu}$ et al. [11] do so.
In recent years, an increasing number of scholars have studied the complex network of stock prices. By constructing a complex network of stock prices, scholars can study the risk contagion, price linkage, and return forecasting among stock prices. In the process of constructing complex networks, many scholars have proposed various methods: Mantegna [12] and Zhuang and Jin [13] proposed a minimum spanning tree algorithm. Pothen et al. [14] used Laplacian graph feature values to construct complex networks; Chi et al. [15] and Peron et al. [16] established stock networks by using the relationship between stock prices as the edges between them in the model. Newman $[17,18]$ proposed the Girvan-Newman (GN) algorithm and fast Newman algorithm to construct complex networks. Zhan et al. [19] proposed the fast unfolding algorithm based on Newman $[17,18]$.

Some of the literature has used the method of complex networks to study the energy financial market. $\mathrm{Xi}$ and $\mathrm{An}$ [20] constructed a complex network using the financial indicators of energy stocks. The study found that the threshold value of 0.7 is the sudden change of the network. With the increase in the threshold value, the community's independence is enhanced. Li et al. [21] used the method of complex networks to study the global energy investment structure and found that the vast majority of foreign investment and foreign investment relations are still in the hands of a few countries.

By analyzing the existing research, we can determine the following. First, the existing research mainly focuses on the study of the interaction between the energy market and other markets. There is little research focus on the mutual contagion of stock risk of different energy companies within the energy industry. Second, in the study of the risk of the energy industry, most of the research mainly analyzes the energy price directly. Few articles use the jump of energy stock prices to conduct in-depth analysis. Third, research on applying complex networks to energy finance risk management is scant. Fourth, when using complex networks to study market risk, few scholars study the dynamic characteristics of complex networks.

Based on the existing research, this paper extracts the jump risk of energy stock prices from high-frequency data of energy stock prices and studies the jump risk of energy industry stock prices. This paper applies the method of complex networks to the energy industry and deeply studies the clustering effect of the jump risk of the energy industry. This paper studies the time-varying characteristics of complex networks and communities of energy price jump risk.

\section{Theoretical Analyses of Realized Jump and Complex Network}

We follow the methods of Zhang et al. [3] to research the time-varying complex network of jump risk in the energy industry. This paper uses the realized method to extract jumps from energy stock volatility and uses it to study the complex network clustering effect within the energy industry. According to the following formula, 
realized volatility $=$ continuous volatility + realized jump .

Equation (1) shows that when we obtain the realized volatility and continuous volatility, we can calculate the realized jump.

3.1. Extracting Volatility by Using the Realized Method. $p_{t, i}$ is the log price of the ith underlying asset on the $t$-th day. The intraday return $r_{t . i}$ can be expressed as

$$
r_{t . i}=p_{t, i}-p_{t, i-1}
$$

We define $R D_{t}$ is the realized variance. The realized variance can be calculated as follows:

$$
R D_{t}=\sum_{i=1}^{N} r_{t, i}^{2}
$$

$N$ is the number of returns on t-th day. By annualizing the realized variance, we can obtain the realized volatility.

$$
R V_{t}=\sqrt{W \cdot R D_{t}},
$$

where $R V_{t}$ is the realized volatility. $W$ is the number of trading days in a year.

3.2. Realized Jump. Early research was based on parametric models to study and estimate jumps by setting specific model forms. With the widespread use of high-frequency data, the use of realized methods to calculate jumps has been favored by scholars. The realized method is nonparametric. It is model-free, convenient to calculate, and accurate to estimate.

The realized jump method was first proposed by Barndoff-Nielsen et al. [22]. Based on quadratic variation theory, they decompose the total variation into two parts: integral volatility and discrete jump.

When the asset return $x(t)$ follows the geometric Brownian motion with a jump, the total volatility of the logarithmic return at time $t$ is not a consistent estimate of the integral volatility. It will be a composite volatility that includes a discrete jump component and continuous volatility components. The quadratic variation of the logarithmic return at time $t, t \in[t, t-1]$ is

$$
Q V_{t}=[r, r]_{t}=\int_{t-1}^{t} \sigma_{s}^{2} d s+\sum_{t-1 \leq s \leq t} \kappa_{s}^{2}, \quad t \in[t, t-1]
$$

According to quadratic variation theory, at time $t$, $t \in[t, t-1]$, the total change in the logarithmic return consists of a continuous integral volatility $\int_{t-1}^{t} \sigma_{s}^{2} \mathrm{~d} s$ $\left(\int_{t-1}^{t} \sigma_{s}^{2} \mathrm{~d} s<\infty\right)$ and a discrete jump part $\sum_{t-1 \leq s \leq t} \kappa_{s}^{2}$.

Anderson and Bollerslev [5] showed that when the frequency of intraday discrete samples is sufficiently large, the quadratic variation consistently estimates the actual volatility. At $t \in[0, T]$,

$$
R V_{t}=\sum_{i=1}^{n} r_{t, i}^{2} \stackrel{n}{\longrightarrow} \longrightarrow \infty Q V_{t}=[r, r]_{t}=\int_{t-1}^{t} \sigma_{s}^{2} d s+\sum_{t-1 \leq s \leq t} \kappa_{s}^{2}, \quad t \in[0, T]
$$

The integral volatility under a continuous path can be estimated from the realized bipower variation (RBV). When the sample is large enough, that is, $n \longrightarrow \infty$, the $\mathrm{RBV}$ is achieved as a consistent estimate of the integral volatility (IV). The RBV is a consistent estimator of integral volatility.

$$
R B V_{t}=u_{1}^{-2}\left(\frac{n}{n-2}\right) \sum_{j=3}^{n}\left|r_{t, j-2} \| r_{t, j}\right|, \quad t \in[0, T] .
$$

$$
u_{a}=E\left[|Z|^{a}\right]=2^{a / 2}\left[\Gamma((a+1) / 2) \Gamma(1 / 2)^{-1}\right] . \quad \Gamma \quad \text { is the }
$$
Gamma function. $Z \sim N(0,1)$. When $a=1$, $u_{1}=E\left[|Z|^{1}\right]=\sqrt{\pi / 2} \cdot n / n-2$ is the correction for the sample space.

3.3. Constructing the Complex Network Based on Prim's Algorithm. There are many different methods to construct a complex network, such as the Kruskal algorithm and the Prim algorithm. After comparing different algorithms, we think that the Prim algorithm has the advantages of stability and simplicity. This paper uses the Prim algorithm to construct the time-varying complex network of the jump of the energy stock prices, which can make the complex network more robust. The closeness to the center is used to analyze the importance of different nodes:

$$
C_{c}\left(v_{i}\right)=\frac{(N-1)}{\left[\sum_{i=1, j \neq 1}^{N} d_{i j}\right]}
$$

Suppose there are $N$ nodes in a complex network. $C_{c}\left(v_{i}\right)$ is the degree of closeness to the center for each node. We use $d_{i j}$ to represent the minimum number of edges between two nodes $i$ and $j$, which belong to $[1, N]$. The greater the degree of closeness of the node, the more importance it has in the network.

3.4. The Community of the Complex Network. After constructing the complex network of the jump of the Chinese energy stock price, we need to further analyze which stock price jump is relatively closely related in the entire complex network. The community is used to study the clustering problem of complex networks. When dividing communities, the fast unfolding algorithm is used to classify the communities of the stocks. 
The modular $Q$ function is a good way to divide the number of communities. The communities can be calculated as follows:

$$
\frac{\sum_{i, j} a_{i j} \delta\left(\sigma_{i}, \sigma_{j}\right)}{\sum_{i, j} a_{i j}}=\frac{\sum_{i, j} a_{i j} \delta\left(\sigma_{i}, \sigma_{j}\right)}{2 M} .
$$

In the modular $Q$ function, we use the community number to distinguish whether two different nodes are in the same community. If the community labels of the two nodes are the same, we consider that the two nodes belong to the same communities. $\sigma_{i}$ represents the community label of node $v_{i}$. If $v_{i}$ and $v_{j}$ are connected, then $a_{i j}=1$. If $v_{i}$ and $v_{j}$ are not connected, then $a_{i j}=0$. If $v_{i}$ and $v_{j}$ belong to the same community. $\delta\left(\sigma_{i}, \sigma_{j}\right)=1$. If they do not belong to the same community, $\delta\left(\sigma_{i}, \sigma_{j}\right)=0 . M$ represents the number of all interconnected edges in the complex network, and $M=\sum_{a_{i j}}$.

The Modular $Q$ function is

$$
Q=\frac{1}{2 M} \sum_{i, j}\left[\left(a_{i j}-\frac{k_{i} k_{j}}{2 M} \delta\left(\sigma_{i}, \sigma_{j}\right)\right)\right] \text {. }
$$

The $i$ and $j$ represent the ith and jth nodes. $K_{i}$ and $k_{j}$ represent the weight of the edge connected to node $i$ and node $j$, respectively. The larger the value of $Q$ is, the better the result of community division is. The fast unfolding algorithm is simple and efficient; thus, we use it to divide the network into communities. The reduction formula of the Modular $Q$ function is

$$
Q=\frac{\sum \text { in }}{2 M}-\left(\frac{\sum \text { tot }}{2 M}\right)^{2}
$$

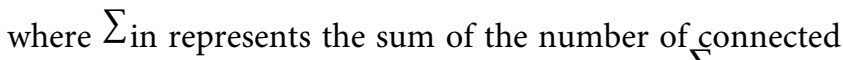
edges of all nodes in the same community, and $\sum_{\text {tot }}$ is the number of edges connected to nodes of the same community.

\section{Empirical Analyses}

4.1. Data. This paper takes all energy stocks listed on China A-Share from October 10, 2016, to October 10, 2018, as an empirical study. We handle the data as follows. (1) Remove the missing values. (2) Use five-minute high-frequency data for the empirical analysis. (3) Assume that the stock price jump is 0 during the suspension period. (4) The data is from the Wind database.

4.2. The Empirical Results of Realized Volatility and Jump of Energy Stock Price. Taking China Petroleum (601857.SH) as an example, the time series of the return of China Petroleum (601857.SH) is shown in Figure 1.

Figure 1 shows that starting from 2018, the yield fluctuated $601857 . \mathrm{SH}$ increased. The volatility and jump risk can be predicted as 601857 .SH will increase significantly starting from 2018. To further explain the basic statistical characteristics of the sample data, Table 1 shows the statistical characteristics of the six stocks.

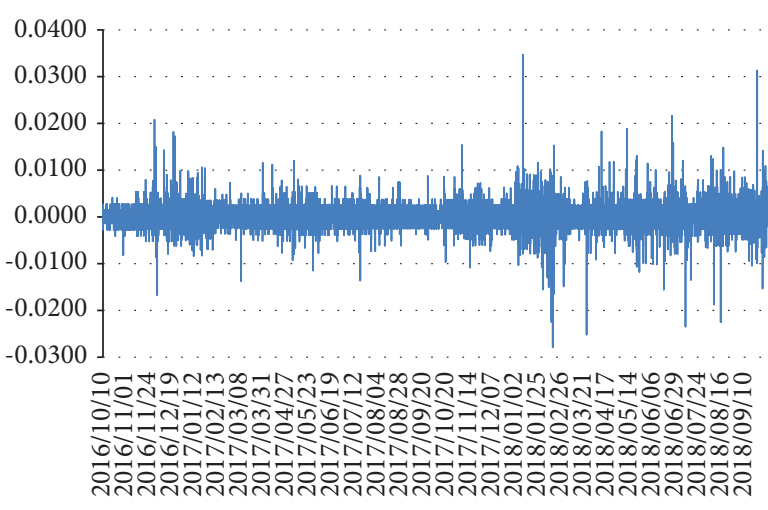

FIGURE 1: 601857.SH 5-minute high-frequency return.

Figure 2 shows the results of the realized volatility and jumps.

Figure 2 shows that the stock price jump is a common phenomenon and an important part of volatility. The trend of 601857.SH in Figure 2 verifies the previous prediction. Beginning in 2018, the realized volatility of 601857 .SH becomes larger, and the frequency and intensity of the jump increase. Figure 2 shows that the stock price jumps in different periods are not completely consistent, and there are certain differences in the frequency and intensity of stock jumps in different time periods. This difference inspires us to study the time-varying characteristics of stock jump risk. For this article, we need to study the time-varying characteristics of complex networks of the energy industry. The statistical properties of the realized volatility and the realized jump are shown in Tables 2 and 3.

4.3. Empirical Results of Time-Varying Complex Network. To study the time-varying characteristics of the complex network of energy stock price jumps, this paper divides the sample interval into four time periods, which are from October 10, 2016, to April 10, 2017; April 11, 2017, to October 10, 2017; October 11, 2017, to April 10, 2018; and April 11, 2018, to October 10, 2018.

4.3.1. Correlation Coefficient Matrix and Distance Matrix of the Energy Stock Jump. Table 4 shows the correlation coefficient matrix for some energy stock price jumps from October 2016 to April 2017.

We use the following formula to transfer the jump correlation coefficient $\rho_{i j}(\Delta t)$ to the corresponding distance $d(i, j)$ :

$$
d(i, j)=\sqrt{2\left[1-\rho_{i j}(\Delta t)\right]}
$$

The smaller the distance $d(i, j)$ is, the stronger the correlation between stocks (Table 5 ).

4.3.2. Using a Distance Matrix to Build an Energy Stock Jump Complex Network. As shown in Figures 3-6, this paper constructs the complex networks of energy stock jumps in four different time periods. Each complex network diagram 
TABLE 1: Statistical characteristic of the sample.

\begin{tabular}{lccccccc}
\hline Stock code & Mean & Maximum & Minimum & Standard deviation & Skewness & Kurtosis & ADF $(P$ value $)$ \\
\hline $601857 . S H$ & $-8.47 E-06$ & 0.0602 & -0.0753 & 0.0030 & 0.0880 & 41.8161 & 0.0001 \\
$601898 . S H$ & $-1.44 E-05$ & 0.0641 & -0.0904 & 0.0032 & 0.1365 & 67.4181 \\
$601918 . S H$ & $-2.69 E-05$ & 0.0892 & -0.0870 & 0.0039 & 1.7818 & 79.0994 & 0.0001 \\
$\ldots$ & $\ldots$ & $\ldots$ & $\ldots$ & $\ldots$ & $\ldots$ & $\ldots .0001$ \\
$000059 . S Z$ & $1.18 E-05$ & 0.0346 & -0.0279 & 0.0020 & 0.3518 & 21.4666 & 0.0001 \\
$000096 . S Z$ & $-2.52 E-06$ & 0.0577 & -0.0392 & 0.0028 & 0.4606 & 24.8307 & 0.0001 \\
$000159 . S Z$ & $-1.29 E-06$ & 0.0719 & -0.0458 & 0.0033 & 0.9072 & 26.7503 & 0.0001 \\
\hline
\end{tabular}

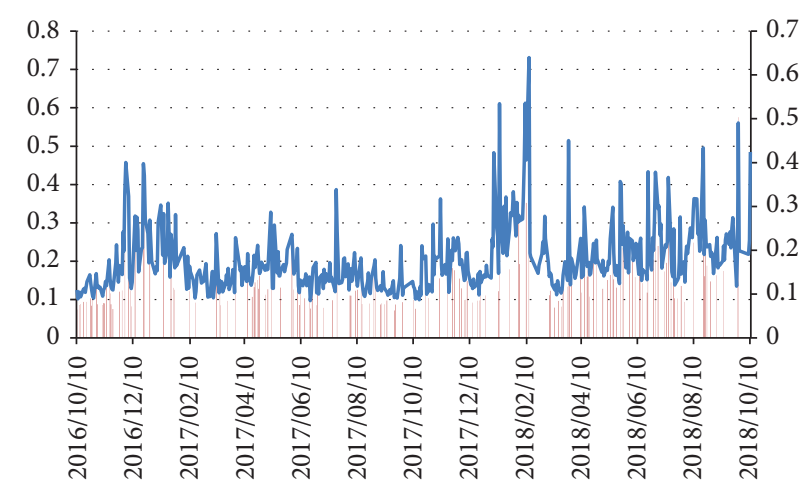

FIGURE 2: Realized volatility and realized jump of 601857.SH.

TABLE 2: Statistical properties of realized volatility for some energy stocks.

\begin{tabular}{lccccccc}
\hline Stock code & Mean & Maximum & Minimum & Standard deviation & Skewness & Kurtosis & ADF $(P$ value $)$ \\
\hline $601857 . S H$ & 0.2028 & 0.7310 & $6.17 E-05$ & 0.0851 & 1.9145 & 8.8743 & $\leq 0.0000$ \\
$601898 . S H$ & 0.2793 & 1.0872 & 0.0001 & 0.1155 & 2.5710 & 13.5124 & $\leq 0.0000$ \\
$601918 . S H$ & 0.3438 & 1.2864 & 0.0000 & 0.1426 & 1.9979 & 9.3526 & $\leq 0.0000$ \\
$\ldots$ & $\ldots$ & $\ldots$ & $\ldots$ & $\ldots$ & $\ldots$ & $\ldots$ & $\ldots .8378$ \\
$000059 . S Z$ & 0.2990 & 1.4772 & 0.0002 & 0.1411 & 2.7712 & 14.5631 & $\leq 0.0000$ \\
$000096 . S Z$ & 0.3120 & 1.6058 & 0.0004 & 0.1690 & 2.8914 & 13.5160 & $\leq 0.0000$ \\
$000159 . S Z$ & 0.3668 & 1.7750 & 0.0002 & 0.2311 & & \\
\hline
\end{tabular}

TABLE 3: Statistical properties of realized jumps for some energy stocks.

\begin{tabular}{lccccccc}
\hline Stock code & Mean & Maximum & Minimum & Standard deviation & Skewness & Kurtosis & ADF $(P$ value $)$ \\
\hline $601857 . S H$ & 0.0471 & 0.5721 & 0.0000 & 0.0824 & 2.3704 & 10.6442 & $\leq 0.0000$ \\
$601898 . S H$ & 0.0604 & 0.7949 & 0.0000 & 0.1051 & 2.6145 & 13.6347 & $\leq 0.0000$ \\
$601918 . S H$ & 0.0823 & 1.0694 & 0.0000 & 0.1354 & 2.3204 & 11.7368 & $\leq 0.0000$ \\
$\ldots$ & $\ldots$ & $\ldots$ & $\ldots$ & $\ldots$ & $\ldots$ & $\ldots .0722$ & 27.6676 \\
$000059 . S Z$ & 0.0488 & 1.1677 & 0.0000 & 0.1179 & 3.4660 & 22.6176 & $\leq 0.0000$ \\
$000096 . S Z$ & 0.0861 & 1.4636 & 0.0000 & 0.1526 & 3.3232 & 17.1421 & $\leq 0.0000$ \\
$000159 . S Z$ & 0.0993 & 1.5125 & 0.0000 & 02004 & & & \\
\hline
\end{tabular}

TABLE 4: Jump correlation coefficient matrix from October 2016 to April 2017.

\begin{tabular}{lccccccc}
\hline & $601857 . S H$ & $601898 . S H$ & $601918 . S H$ & $\ldots$ & $000059 . S Z$ & $000096 . S Z$ & $000159 . S Z$ \\
\hline $601857 . S H$ & 1.0000 & 0.3860 & 0.2683 & $\ldots$ & 0.4625 & 0.3234 & 0.3725 \\
$601898 . S H$ & 0.3860 & 1.0000 & 0.2589 & $\ldots$ & 0.2664 & 0.2192 \\
$601918 . S H$ & 0.2683 & 0.2589 & 1.0000 & $\ldots$ & 0.1162 & 0.0752 & 0.4458 \\
$\ldots$ & $\ldots$ & $\ldots$ & $\ldots$ & $\ldots$ & $\ldots$ & $\ldots .0973$ \\
$000059 . S Z$ & 0.4625 & 0.3234 & 0.3725 & $\ldots$ & 1.0000 & 0.2631 & 0.2270 \\
$000096 . S Z$ & 0.2664 & 0.2192 & 0.4458 & $\ldots$ & 0.2631 & 1.0000 \\
$000159 . S Z$ & 0.1162 & 0.0752 & 0.0973 & $\ldots$ & 0.2270 & 0.1187 \\
\hline
\end{tabular}


TABle 5: Jump distance matrix from October 2016 to April 2017.

\begin{tabular}{lccccccc}
\hline & $601857 . S H$ & $601898 . S H$ & $601918 . S H$ & $\ldots$ & $000059 . S Z$ & $000096 . S Z$ & $000159 . S Z$ \\
\hline $601857 . S H$ & 0.0000 & 1.1081 & 1.2097 & $\ldots$ & 1.0369 & 1.2113 \\
$601898 . S H$ & 1.1081 & 0.0000 & 1.2175 & $\ldots$ & 1.1632 & 1.2497 & 1.3295 \\
$601918 . S H$ & 1.2097 & 1.2175 & 0.0000 & $\ldots$ & 1.1203 & 1.0528 & 1.34360 \\
$\ldots$ & $\ldots$ & $\ldots$ & $\ldots$ & $\ldots$ & $\ldots$ & $\ldots$ & $\ldots .0000$ \\
$000059 . S Z$ & 1.0369 & 1.1632 & 1.1203 & $\ldots$ & 1.2140 & 1.2434 \\
$000096 . S Z$ & 1.2113 & 1.2497 & 1.0528 & $\ldots$ & 1.2140 & 0.0000 \\
$000159 . S Z$ & 1.3295 & 1.3600 & 1.3436 & $\ldots$ & 1.2434 & 1.3277 & 0.0000 \\
\hline
\end{tabular}

has 62 nodes, which represent the jump of 62 energy sample stock prices. In complex network diagrams, the number of interconnected nodes and the length of the distance between nodes are used to represent complex network relationships. The more nodes that are connected to each other, the greater the stock price jumps directly when the node's energy stock price jumps. The distance between the nodes represents the weight between the nodes. If the distance between the two nodes is shorter, then the correlation between the jumps of the stock price is stronger. If the node does not appear in the complex network diagram, then there is no correlation between the jump of the energy stock price and other stock prices.

We can draw the following conclusions. (1) China's energy stock prices directly or indirectly affect one another. Figures 3-6 show that the 62 sample points in this paper all appear in the complex network diagram. They will be directly or indirectly linked, indicating that the jump risk of China's energy stock price is ubiquitous and can be influenced by each node. (2) In the complex network diagram, the influence of different energy stock price jumps is inconsistent. For example, in Figure 3, node 55 is directly connected to multiple stocks. When the stock price of this node jumps, it will cause node 13 . The stock prices on 15, 15, 22,32 , and 37 jumped, but the stocks directly connected to nodes 6 and 36 were fewer. The results show that in the energy stock industry, the contagion of risks in different stocks is inconsistent. (3) The infectious power of the energy stock jump risk will change with time. Comparing node 55 in Figure 3 with node 31 in Figure 5 shows that there are 6 nodes in Figure 3. Nodes 55 are connected, but only one node in Figure 5 is connected to node 55. In Figure 5, a total of 8 nodes are connected to node 31 , indicating that the riskinfecting ability of the same source-only stock changes over time in the entire network diagram. (4) The shape of the complex network changes with time, indicating that the energy stock price jumps the complex network relationship over time. Figures 3 and 4 show the complex network of energy stock hopping before October 2017, showing a "banded" distribution. There are fewer nodes directly adjacent to each node, indicating that the energy stock price jumps on a certain node. It is not easy to directly cause a large jump in the energy stock price of the entire network. However, Figures 5 and 6 show that the complex network of energy stock hopping after October 2017 tends to be "grouped", and the nodes directly adjacent to each node increase, indicating that the energy stock price occurs on a certain node. Jumping will directly cause more energy stock prices to jump. The results show that starting from October 2017, the correlation between stock market jump risks in the energy industry is stronger, and the risk contagion between energy stocks is stronger.

4.3.3. Analysis of the Importance of Network Nodes. This study uses closeness centrality to analyze the importance of the nodes. Table 6 shows that the energy stocks with the highest centrality value in the complex network diagrams of four different time periods are 300157. SZ, 000059. SZ, 000059. SZ, and 000968. SZ, respectively.

Table 6 shows the following. (1) The role of each node in the complex network on the stability and invulnerability of the network is inconsistent. In the period of October 2016 to April 2017, 300157.SZ is most important to the stability of the network. (2) The importance of nodes in complex networks is time-varying. In different time periods, according to the results of the closeness centrality, the top five energy stock codes are not completely consistent. (3) For the Chinese energy market, the coal industry is the most important throughout the network tree. The analysis shows that in these four time periods, more than $50 \%$ of the stocks are coal stocks, and after October 2017, the proportion of coal stocks increased. The results show that the stability of the coal industry is very important for the development of China's energy industry. The reason is that China is a large country in coal production and consumption. In China's energy consumption structure, coal energy consumption stands at more than $65 \%$. Therefore, at present, the coal industry is at the core of China's energy finance complex network.

4.3.4. Complex Network Dynamic Cluster Analysis. The previous analysis shows that the time-varying characteristics of the complex network of energy stock jumps are obvious. To further explain the dynamic clustering characteristics of energy stock jumping complex networks, a fast unfolding algorithm is used.

The results of the community-based Q-based community classification in Table 7 show that from October 2016 to October 2018, the optimal number of community divisions is gradually reduced, indicating that more energy stock price jumps are included in the same community. The clustering characteristics within the community are more obvious, and the results further confirm that after October 2017, the contagion of the risk of jumping between energy stocks becomes stronger. The clustering analysis results of complex 


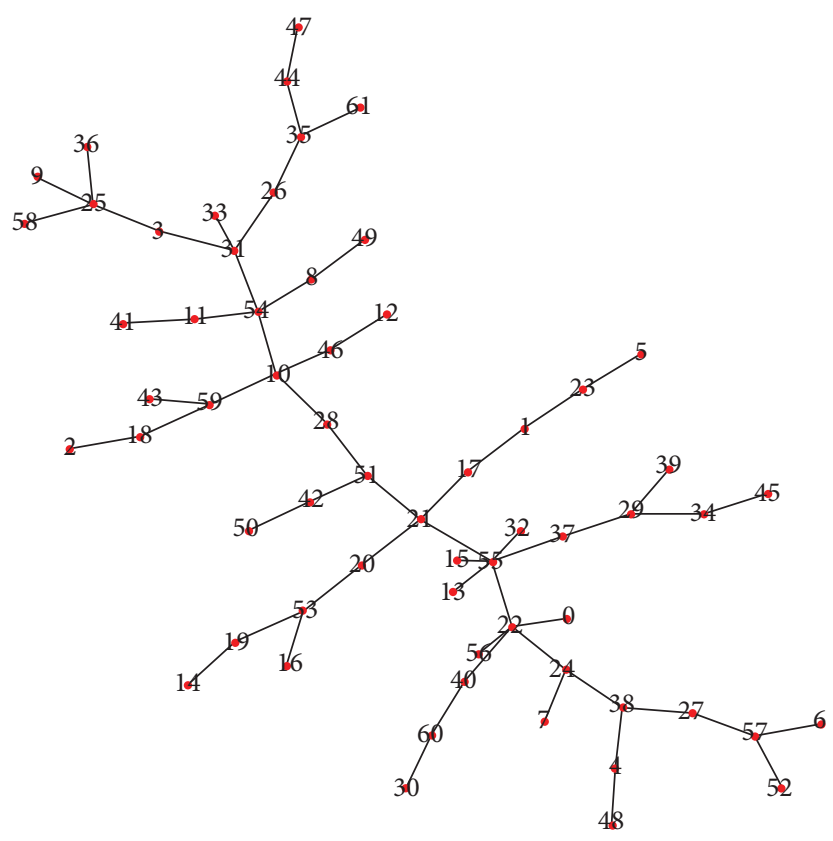

Figure 3: Energy stock jump complex network from October 2016 to April 2017.

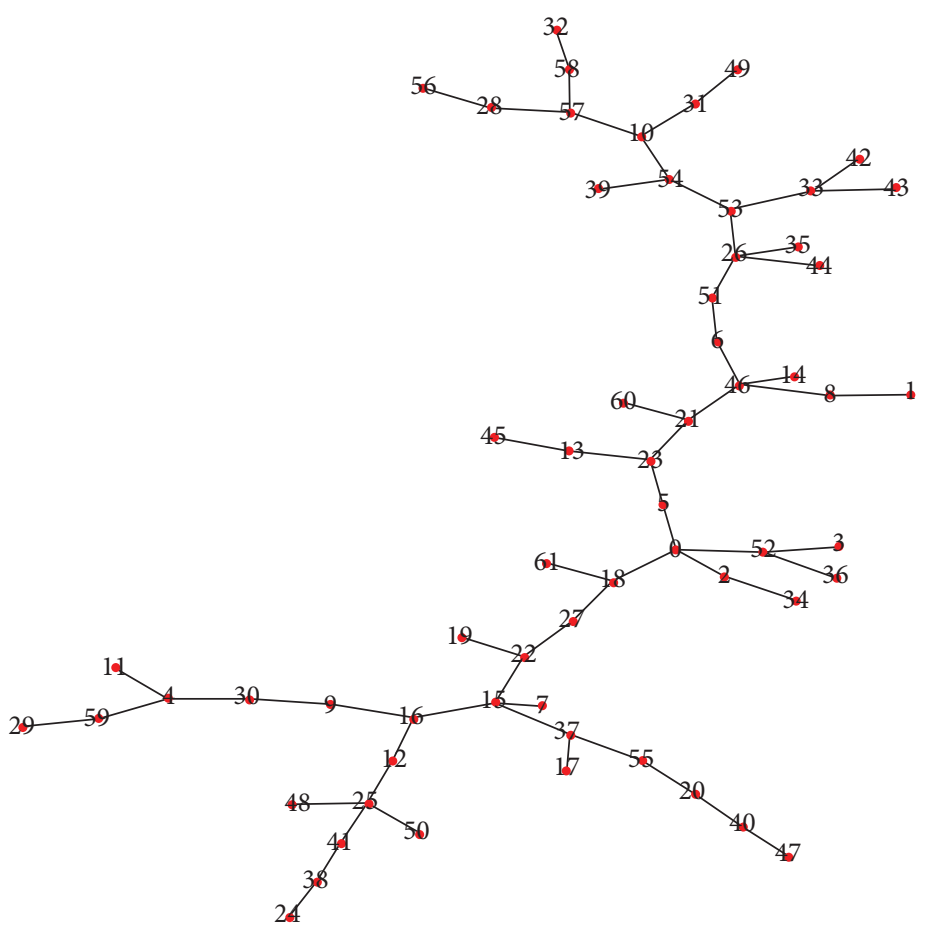

Figure 4: Energy stock jump complex network from April 2017 to October 2017.

networks in different time periods are reported in Figures 7-10. The different colors in Figures 7-10 represent different communities. The jump correlation between communities is shown in Table 8 .

The analysis of the average correlation coefficient of the communities in different time periods reported in Table 8 shows that the energy enterprises with the highest average correlation coefficient in different time periods are inconsistent, and the time-varying characteristic is obvious. Prior to October 2017, most of the energy stocks in the communities with the highest average correlation coefficient were energy mining equipment manufacturers, but after October 2017, most of the energy stocks in the communities with the highest average correlation coefficient were coal companies and new energy companies. The empirical results show that the degree of risk contagion of 


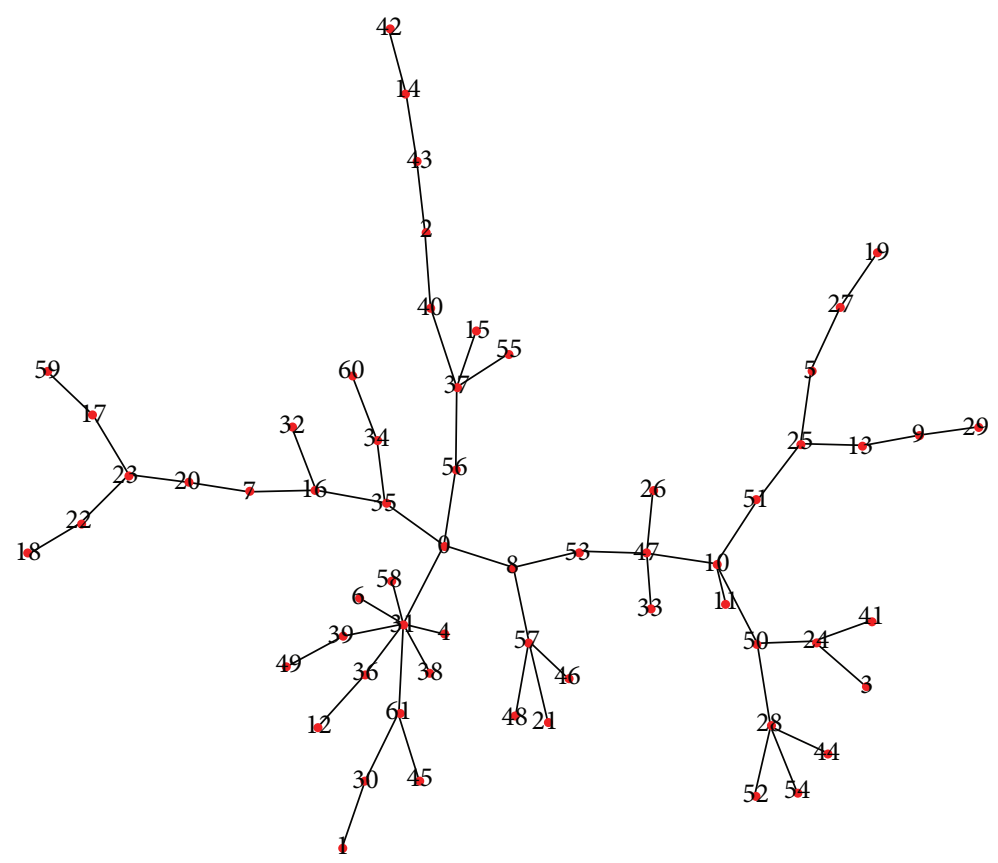

Figure 5: Energy stock jump complex network from October 2017 to April 2018.

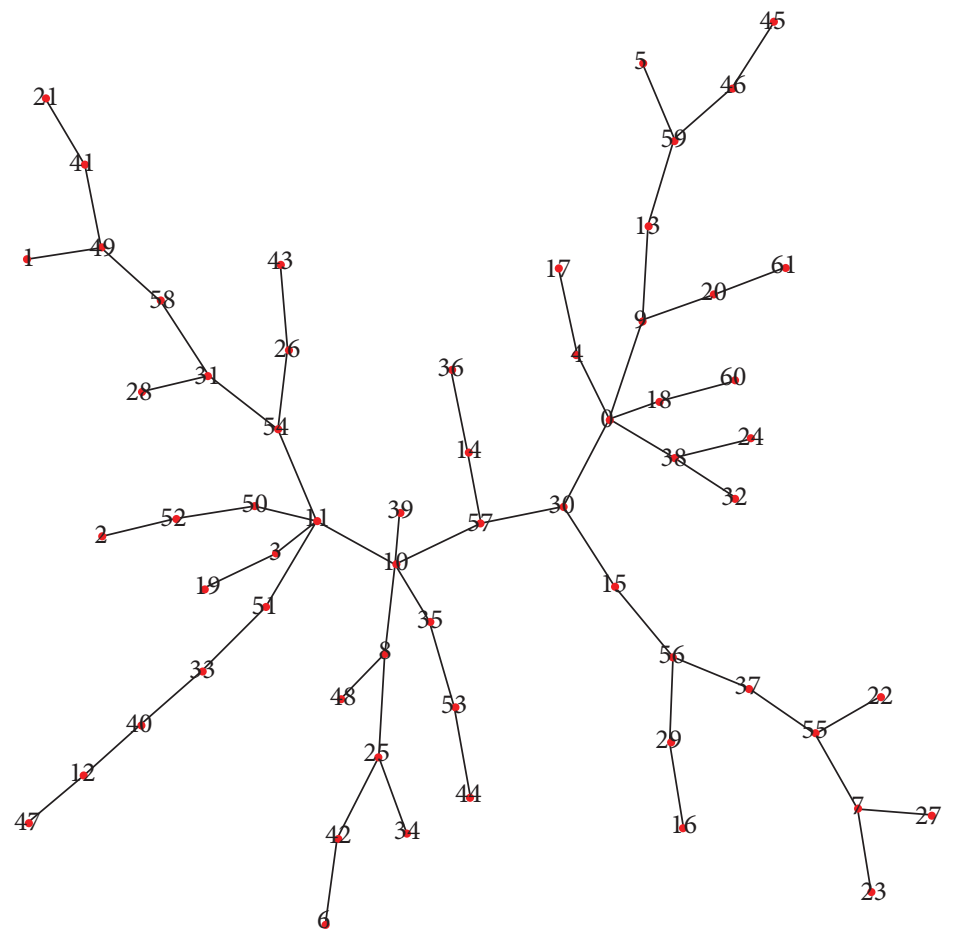

Figure 6: Energy stock jump complex network from April 2018 to October 2018.

different types of energy companies is inconsistent at different time periods. China must pay attention to the risk contagion within the coal industry and the new energy industry. China's coal stock jump risk is highly correlated and prone to risk contagion within the coal industry. Once the risk accumulates within the coal industry, it is likely to affect the entire energy industry in China. China also needs to pay attention to the risk contagion problem within new energy enterprises. At present, many enterprises in China develop mainly new energy focused on electricity, solar energy, wind energy, and so on. These enterprises have similar development paths and are prone to systemic risks, which hinder the development of the new energy industry in China. 
TABLE 6: Importance analysis of complex network nodes based on closeness centrality.

\begin{tabular}{lcccc}
\hline Ranking & $2016.10-2017.04$ & $2017.04-2017.10$ & $2017.10-2018.04$ & $2018.04-2018.10$ \\
\hline 1 & $300157 . S Z$ & $000059 . S Z$ & $000059 . S Z$ & $000968 . S Z$ \\
2 & $601101 . S H$ & $000723 . S Z$ & $000937 . S Z$ & $601898 . S H$ \\
3 & $601808 . S H$ & $002629 . S Z$ & $601666 . S H$ & $600339 . S H$ \\
4 & $600188 . S H$ & $300191 . S Z$ & $600348 . S H$ & $002128 . S Z$ \\
5 & $000983 . S Z$ & $600157 . S H$ & $600508 . S H$ & $000059 . S Z$ \\
\hline
\end{tabular}

TABLe 7: Modular Q statistics.

\begin{tabular}{lcc}
\hline Period & Optimal number of communities & Modular Q value \\
\hline $2016.10-2017.04$ & 10 & 0.7846 \\
$2017.04-2017.10$ & 9 & 0.7698 \\
$2017.10-2018.04$ & 8 & 0.7802 \\
$2018.04-2018.10$ & 7 & 0.7740 \\
\hline
\end{tabular}
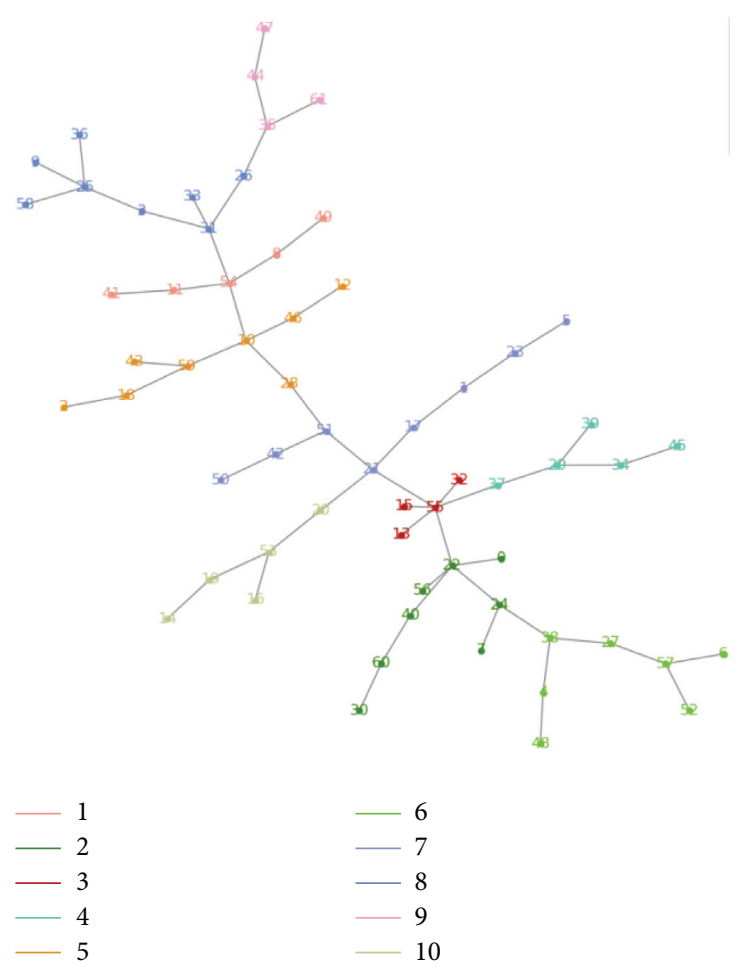

FIgURE 7: The community of energy stock price jump from October 2016 to April 2017. 


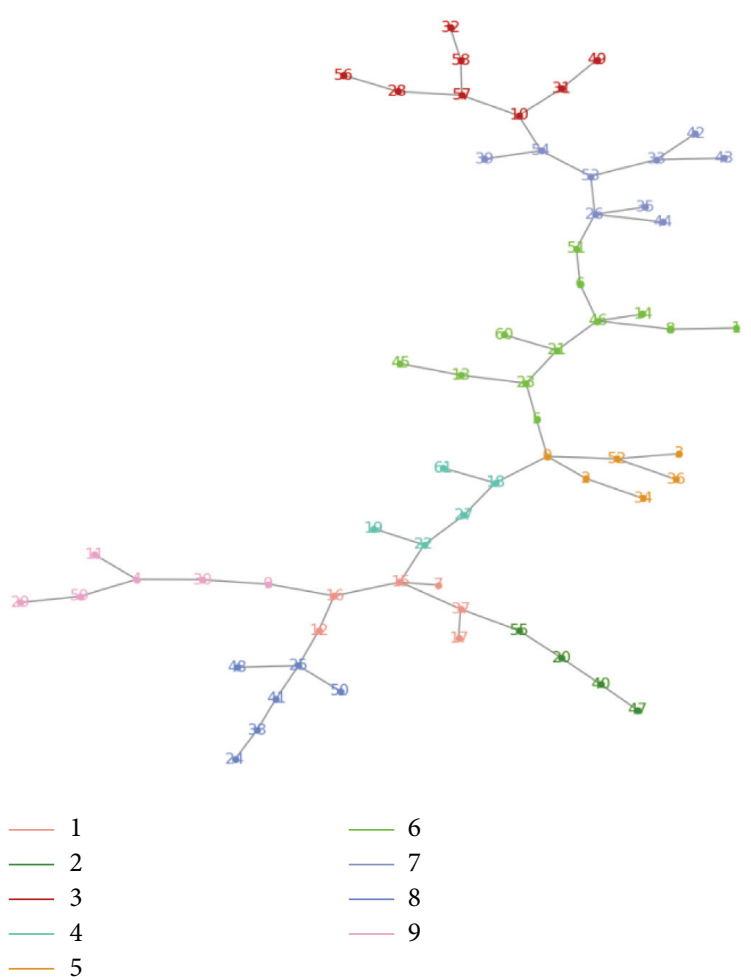

FIgURE 8: The community of energy stock price jump from April 2017 to October 2017.

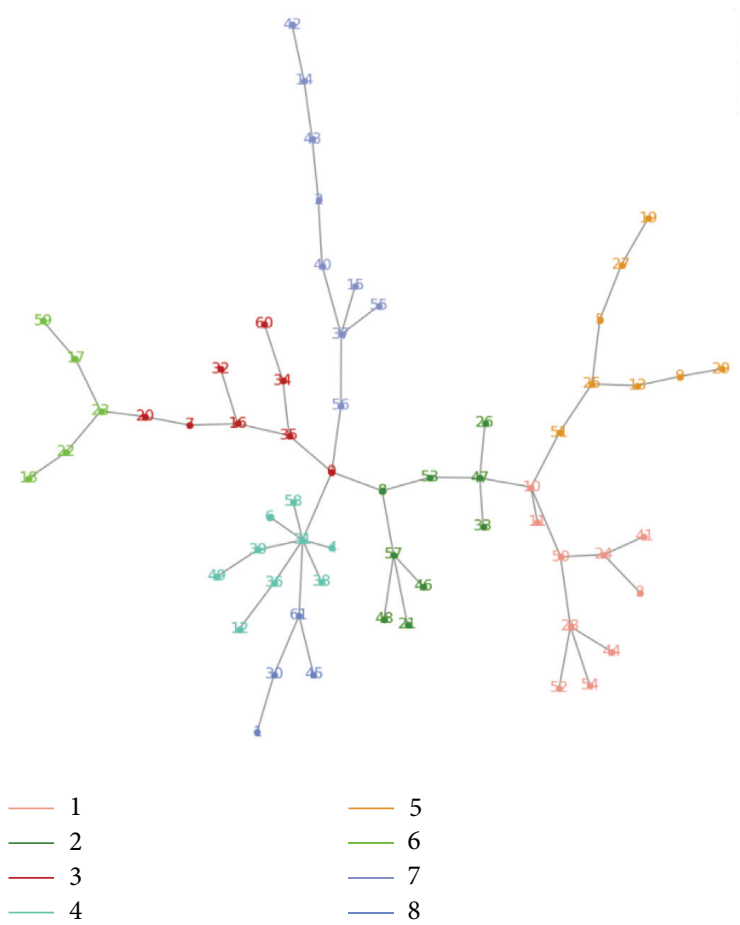

Figure 9: The community of energy stock price jump from October 2017 to April 2018. 


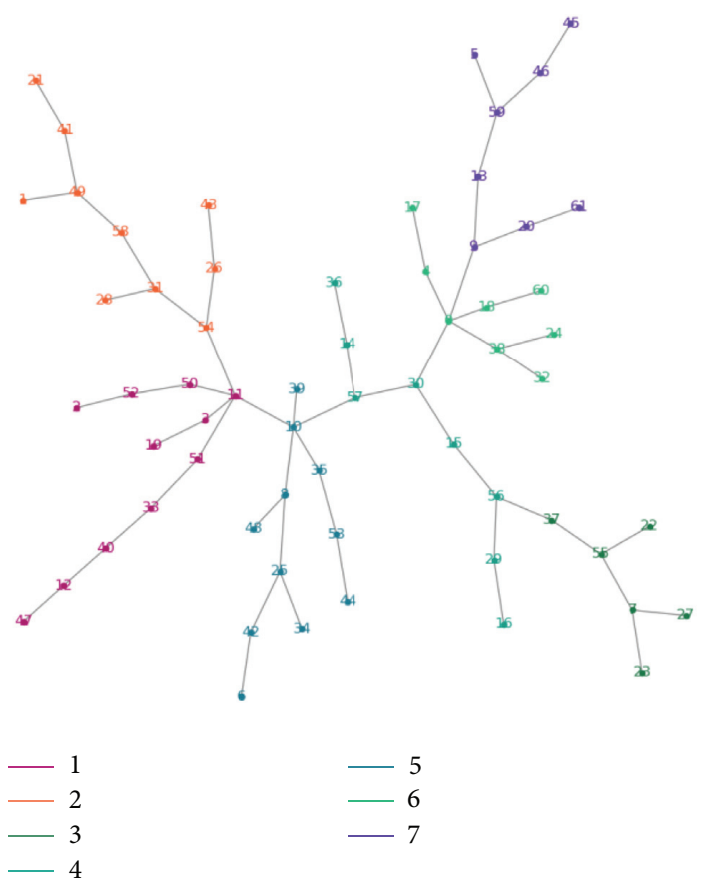

FIgURE 10: The community of energy stock price jump from April 2018 to October 2018.

TABLE 8: Communities with the strongest correlation coefficient at different time periods.

\begin{tabular}{|c|c|c|}
\hline Period & Stock code & Average correlation coefficient \\
\hline $\begin{array}{l}2016.10- \\
2017.04\end{array}$ & 600387.SH, 002353.SZ, 002267.SZ, 601808.SH & 0.4855 \\
\hline $\begin{array}{l}2017.04- \\
2017.10\end{array}$ & 600583.SH, 000852.SZ, 002221.SZ, 002353.SZ, 002490.SZ, 002554.SZ & 0.4038 \\
\hline $\begin{array}{l}2017.10- \\
2018.04\end{array}$ & $\begin{array}{r}\text { 000552.SZ, 600777.SH, 000983.SZ, 002128.SZ, 600971.SH, 601088.SH, 601225.SH, } \\
\text { 601699.SH, 600028.SH, 600188.SH }\end{array}$ & 0.6198 \\
\hline $\begin{array}{l}2018.04- \\
2018.10\end{array}$ & $\begin{array}{c}\text { 000096.SZ, 601918.SH, 600856.SH, 601015.SH, 300157.SZ, 601699.SH, 600123.SH, } \\
\text { 600188.SH, 600348.SH }\end{array}$ & 0.5152 \\
\hline
\end{tabular}

\section{Conclusions}

This paper systematically studies the clustering problem of dynamic complex networks of stock jumping risk of energylisted companies. Based on the research of this paper, we conclude the following. (1) The jump of the stock price is an important component of volatility. (2) The complex network of stock price jumps of energy enterprises has obvious timevarying characteristics. After October 2017, the risk contagion of the stock price jump is more likely to occur. (3) The community of the energy stock price complex network also has strong time-varying characteristics. (4) The coal industry is the most critical in the complex network of the entire energy industry, and the internal risk of the coal industry is highly contagious. (5) The risk contagion within the new energy industry community is key for the development of the new energy industry.

Based on the above conclusions, we believe that in the field of energy finance, the following points should be considered. (1) The energy stock price jump is a common phenomenon, but the infection problem of stock price jump risks needs specific analysis. (2) The issue of energy financial risk management should be viewed holistically. (3) There are different ways to jump risk contagion in the energy industry. We need to systematically analyze the clustering results of the energy industry, penetrate specific industries according to the results, and adopt appropriate methods for risk management. (4) China needs to take measures to protect the jumping risk of the coal industry.

\section{Data Availability}

All data used to support the finding of the study are available from the corresponding author upon request.

\section{Conflicts of Interest}

The authors declare that they have no conflicts of interest.

\section{Acknowledgments}

This paper was supported by the 2020 Suqian Science and Technology Planning Project (Social Development): "Research on Science and Technology Finance Boosting the Development of Science and Technology SMEs in Suqian under the Background of the Epidemic" (S202005), the 
Zhejiang Science and Technology Information Research Institute R\&D Project: "A Data Processing Method for Intelligent Mining and Text Anomaly Monitoring" (R2021D001), and Doctoral Research Initiation Project Fund (2019BSXM11).

\section{References}

[1] T. Kaneko and B. S. Lee, "Relative importance of economic factors in the U.S. And Japanese stock markets," Journal of the Japanese and International Economies, vol. 9, no. 3, pp. 290-307, 1995.

[2] S. A. Basher and P. Sadorsky, "Oil price risk and emerging stock markets," International Finance, vol. 17, no. 2, pp. 224-251, 2004.

[3] Y. J. Zhang, Y. Fan, H. T. Tsai, and Y. M. Wei, "Spillover effect of US dollar exchange rate on oil prices," Journal of Policy Modeling, vol. 30, no. 6, pp. 973-991, 2008.

[4] P. K. Narayan, S. Narayan, and X. Zheng, "Gold and oil futures market: are markets efficient?" Applied Energy, vol. 87, no. 10, pp. 3299-3303, 2010.

[5] T. G. Andersen and T. Bollerslev, "Deutsche mark-dollar volatility: intraday activity patterns, macroeconomic announcements, and longer run dependencies," The Journal of Finance, vol. 53, no. 1, pp. 219-265, 1998.

[6] O. E. Barndorff-Nielsen and N. Shephard, "Econometric analysis of realized Co-variation: high frequency based covariance, regression, and correlation in financial economics," Econometrica, vol. 72, no. 3, pp. 885-925, 2004.

[7] C. F. Wang, Y. Ning, Z. M. Fang, and Y. Li, "An empirical research on jump behavior of realized volatility in Chinese stock markets," Systems Engineering, vol. 26, no. 2, pp. 1-6, 2008.

[8] T. G. Andersen, T. Bollerslev, and X. Huang, "A reduced form framework for modeling volatility of speculative prices based on realized variation measures," Journal of Econometrics, vol. 160, no. 1, pp. 176-189, 2011.

[9] Z. F. Zeng and J. Zuo, "Research on relationship of jump and volatility spillover between shanghai and Hong Kong stock market returns - - based on SVCJ model by MCMC algorithm," Chinese Journal of Management Science, vol. 21, no. S1, pp. 334-340, 2013.

[10] W. J. Xu, F. Ma, and Y. Wei, "Investigating the performance of the high-frequency volatility models using the nonparametric jump tests," Systems Engineering, vol. 34, no. 12, pp. 10-16, 2016.

[11] S. Hu, Z. Gu, Y. Wang, and X. Zhang, "An analysis of the clustering effect of a jump risk complex network in the Chinese stock market," Physica A: Statistical Mechanics and its Applications, vol. 523, pp. 622-630, 2019.

[12] R. N. Mantegna, "Hierarchical structure in financial markets," The European Physical Journal B - Condensed Matter and Complex Systems, vol. 11, no. 1, pp. 193-197, 1999.

[13] X. W. Zhuang and X. Jin, "The research of correlation between network topological index and volatility of shanghai stock market," Journal of Donghua University, vol. 36, no. 3, pp. 453-456, 2015.

[14] A. Pothen, H. D. Simon, and K. P. Liou, "Partitioning sparse matrices with eigenvectors of graphs," SIAM Journal on Matrix Analysis and Applications, vol. 11, no. 3, pp. 430-452, 1990.

[15] K. T. Chi, J. Liu, and F. C. M. Lau, "A network perspective of the stock market," Journal of Empirical Finance, vol. 17, no. 4, pp. 659-667, 2010.
[16] T. K. Peron, L. F. Costa, and F. A. Rodrigues, "The structure and resilience of financial market networks," Chaos An Interdisciplinary Journal of Nonlinear Science, vol. 22, no. 1, p. 193, 2012.

[17] M. E. J. Newman, "Fast algorithm for detecting community structure in networks," Physical Review E-Statistical, Nonlinear and Soft Matter Physics, vol. 69, no. 6, Article ID 066133, 2003.

[18] M. E. J. Newman and M. Girvan, "Finding and evaluating community structure in networks," Physical Review E-Statistical, Nonlinear and Soft Matter Physics, vol. 69, no. 2, Article ID 026113, 2004.

[19] W. W. Zhan, J. L. Xi, and Z. X. Wang, "Hierarchical agglomerative community detection algorithm based on similarity modularity," Journal of System Simulation, vol. 29, no. 5, pp. 1028-1040, 2017.

[20] X. Xi and H. An, "Research on Energy Stock Market Associated Network Structure Based on Financial indicators," Physica A Statistical Mechanics and its Applications, vol. 490, pp. 1309-1323, 2018.

[21] H. Li, H. An, F. Wei, Y. Wang, W. Zhong, and L. Yan, "Global energy investment structure from the energy stock market perspective based on a Heterogeneous Complex Network Model," Applied Energy, vol. 194, pp. 648-657, Article ID S0306261916306596, 2016.

[22] O. E. Barndorff-Nielsen, B. Nielsen, N. Shephard, and C. Ysusi, "Measuring and Forecasting Financial Variability Using Realised Variance," State Space and Unobserved Component Models, pp. 205-235, 2004. 